\title{
Rescate de un acceso vascular para hemodiálisis. A propósito de un caso
}

\author{
Isabel Crehuet Rodríguez, María Albina Bernárdez Lemus, Beatriz Toribio Manrique, María del Mar Sánchez \\ Arribas, Raquel Villa Ruíz, Marta Ramírez Crehuet
}

Unidad de Diálisis. Hospital Universitario Río Hortega. Valladolid. España

\section{Resumen}

El mejor acceso vascular (AV) para hemodiálisis (HD) es, sin duda, la fístula arteriovenosa interna nativa (FAVI) ya que presenta más ventajas y menos complicaciones que otros accesos vasculares.

Enfermería, gracias a los protocolos de vigilancia del $A V$, juega un papel fundamental en la detección temprana de las complicaciones que puedan llevar a la pérdida del mismo.

Realizamos este estudio con dos objetivos:

1) Dar a conocer un caso clínico de una FAVI en el que aparecieron sucesivamente varias complicaciones.

2) Analizar la importancia que tiene la existencia de un equipo multidisciplinar para los accesos vasculares, tanto en la realización y seguimiento del mismo como en el tratamiento de las complicaciones que pudieran aparecer.

Se estudia a un paciente varón con enfermedad renal crónica estadio V, portador de una FAVI radiocefálica derecha que, en una inspección rutinaria del AV cuando acude a la sesión de HD, enfermería detecta disminución de soplo y thrill apareciendo primero una trombosis, seguida de una estenosis tratada quirúrgicamente y finalmente una grave infección en la herida quirúrgica por un germen Gram negativo. A lo largo del estudio se van presentando las sucesivas complicaciones que surgieron y las estrategias desarrolladas por los miembros del equipo multidisciplinar: enfermeras, nefrólogos y

Correspondencia:
Isabel Crehuet Rodríguez
Hospital Universitario Río Hortega. Unidad de Diálisis
C/ Dulzaina 2, 47012 Valladolid
Email: crebel@hotmail.com

cirujanos vasculares, entre otros, para solventarlas, lo cual finalmente se consiguió llegando a rescatar un acceso vascular que, a priori, dábamos por perdido.

PALABRAS CLAVE

- FÍSTULA ARTERIOVENOSA INTERNA

- EQUIPO MULTIDISCIPLINAR

- INFECCIÓN FAVI

- HEMODIÁLISIS

Rescuing a vascular access for hemodialysis. Report of a case

\section{Abstract}

The best vascular access (VA) for hemodialysis (HD) is undoubtedly the native arteriovenous fistula (AVF) as presents more advantages and fewer complications than other vascular access.

Nursing, using surveillance protocols of vascular, plays an essential role in the early detection of complications that might lead to their loss.

We conducted this study with two objectives:

1) To present a clinical case of an AVF in which appeared on several complications.

2) Analyze the importance of the existence of a multidisciplinary team for vascular access in the implementation and monitoring of the same and in the treatment of possible complications.

A male patient in stage five of CKD is studied, bearing a right radiocephalic $A V F$, which during a routine inspection of the AV when he came to the HD session, 
nursing detected decreased murmur and thrill appearing thrombosis first followed by stenosis treated surgically and finally a serious infection in the surgical wound by a Gram negative germs. During the study, subsequent complications that emerged and the strategies developed by the members of the multidisciplinary team are being presented: nurses, nephrologists and vascular surgeons, among others, to solve them, which finally got coming to rescue a vascular access a priori lost.

\section{KEYWORDS}

\section{- ARTERIOVENOUS FISTULA \\ - MULTIDISCIPLINARY TEAM \\ - AVF INFECTION \\ - HEMODIALYSIS}

\section{Introducción}

El acceso vascular (AV) es un requisito imprescindible y necesario para realizar hemodiálisis (HD); está directamente relacionado con la morbimortalidad del paciente y su calidad de vida. EI mejor AV es, sin duda y tal como recomiendan todas las guías ${ }^{1,2}$ la fístula arteriovenosa interna nativa (FAVI) por las ventajas que presenta: fácil accesibilidad, menores costes de realización y mantenimiento, mayor y mejor supervivencia, menor número de ingresos hospitalarios debido principalmente a su menor número de complicaciones respecto a los otros posibles accesos: fístulas arteriovenosas protésicas (FAVp) y catéteres venosos centrales tunelizados (CVCT).

Sin embargo, aunque se presenten en menor número, varias son las complicaciones que pueden aparecer: estenosis, trombosis, infecciones, aneurismas, etc. siendo las dos primeras las más frecuentes ${ }^{1}$.

Dentro del equipo multidisciplinar del AV, enfermería juega un papel fundamental en la detección temprana de dichas complicaciones, gracias a los protocolos de vigilancia del acceso vascular (inspección, palpación, auscultación, etc.) en cada sesión, para evitar en muchas ocasiones, su pérdida y todos los inconvenientes que ello conlleva: implantación de catéteres venosos centrales, realización de una nueva FAVI, etc.

Los objetivos de nuestro estudio son:

- Presentar un caso clínico en el que fueron apareciendo sucesivamente varias complicaciones: trombosis, estenosis e infección de una FAVI.
- Analizar la importancia de un equipo multidisciplinar en el seguimiento y tratamiento de dichas complicaciones.

\section{Paciente y Método}

Paciente varón de 79 años de edad, con antecedentes de:

Hipertensión arterial desde 1980.

Enfermedad renal crónica (ERC) diagnosticada en 1984, por probable glomerulonefritis crónica y agenesia renal izquierda congénita, con riñón derecho pélvico (no biopsiado), que evolucionó a ERC estadio 5, precisando HD de forma crónica desde septiembre de 2008.

Anemia e hiperparatiroidismo secundarios a ERC.

Patología cardiaca: hipertrofia ventricular izquierda con insuficiencia tricuspídea.

Linfoma no Hodking difuso de células grandes en 2005, tratado con quimioterapia y radioterapia, desde hace años en remisión completa.

Hipotiroidismo primario desde enero de 2008.

Trasplante renal de cadáver en 2014; se coloca un riñón invertido en fosa ilíaca izquierda. El post-trasplante inmediato se complicó con necrosis tubular aguda severa, linfocele e infección urinaria por Pseudomona aeruginosa y Enterococcus faecium, no consiguiéndose diuresis eficaz en ningún momento, precisando continuar con tratamiento de HD.

Respecto a los accesos vasculares:

FAVp humerocefálica que se trombosó. Se realizó una nueva FAVI radiocefálica derecha que es el objeto de este estudio.

\section{Análisis del caso}

El 25/09/2014 antes de iniciar la sesión de HD, enfermería detecta una disminución importante del soplo y del thrill. Tras la realización de una ecografía se objetiva trombosis parcial del acceso, por lo que se envía a cirugía vascular. Al día siguiente, se realiza trombectomía para poder obtener flujos adecuados en HD. Durante unas horas presenta sangrado leve de herida quirúrgica, por lo que se mantiene Clexane $60 \mathrm{mg} / 24 \mathrm{~h}$. No se reinicia Sintrom. 
El 27/09/2014 al realizar inspección y palpación de FAVI se observa ausencia de sangrado, ligero edema y pequeño hematoma. Se realiza Eco-Doppler de FAVI y se detecta la presencia de un pequeño hematoma y una estenosis dudosa; a lo largo del trayecto de la vena cefálica se observa buen calibre; con el Doppler pulsado se aprecia buen flujo. Se decide pinchar la arteria a 5-6 cm de la anastomosis y la vena unos centímetros más proximal, a la altura de la flexura del codo, para hacer la sesión de $H D$, pero, aunque han mejorado los flujos, éstos no son adecuados (oscilan entre $250-270 \mathrm{ml} / \mathrm{min}$ ), por lo que tres días después se realiza una fistulografía con abordaje a través de la arteria humeral derecha y se observa una estenosis del $50 \%$ en la salida de la vena eferente, que se divide tempranamente en dos ramas con buen diámetro. Tras este diagnóstico, el día 6 de octubre, se repara quirúrgicamente la FAVI mediante desconexión y reanastomosis del tramo arterial a una de estas venas eferentes.

Al día siguiente (7-10-2014) se realiza HD con punción de la vena reanastomosada, durante 2 horas, a pesar de que apenas tiene soplo y thrill, por lo que se decide la implantación de un CVCT para tener un acceso vascular más seguro, hasta ver evolución de FAVI. El catéter se coloca al día siguiente, sin complicaciones iniciales. Posteriormente comienza con sangrado a través del orificio de salida del catéter (OSC) y de herida de FAVI, la cual necesita compresión y vendaje con control. Se suspende anticoagulación valorando riesgo-beneficio. Se administra Vancomicina vía parenteral por el riesgo de infección ante la intervención quirúrgica sobre FAVI y por el sangrado que, aunque leve, es persistente.

A los nueve días de la intervención, se observa gran hematoma en FAVI por lo que se pone un apósito de Linitul más vendaje y se envía a Cirugía Vascular al hospital. Una vez valorado, se retiran suturas, se toma muestra para cultivo, se limpia la herida (Fig. 1) y se pautan curas con suero salino al $0,9 \%$ más solución de povidona yodada (Betadine ${ }^{\circledR}$ ), dejando punta de gasa a modo de drenaje.

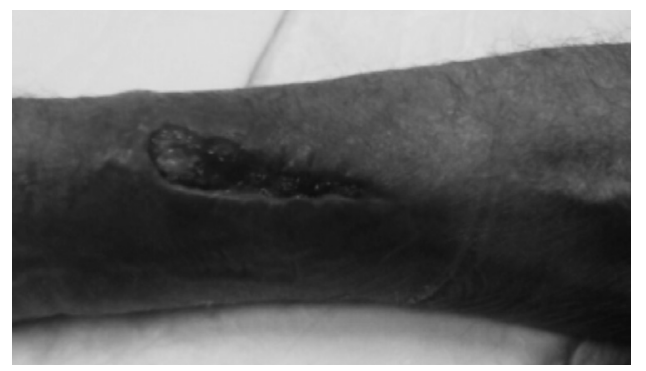

Fig. 1. Herida limpia una vez retiradas las suturas.
Después de dos curas comenzamos con una cura húmeda con apósitos hidrocoloides con plata iónica (Aquacel $\mathrm{Ag}^{\circledR}$ ), ya que la herida tenía mal aspecto. En el exudado cultivado apareció un Staphylococcus epidermidis que, según el laboratorio de Microbiología, podría considerarse como flora saprofita normal, sin embargo, la herida en la siguiente cura presentaba aún peor aspecto, llegando a tener unas dimensiones de $9 \mathrm{~cm} \times 4 \mathrm{~cm} \times$ $0,3-0,8 \mathrm{~cm}$ de profundidad aproximadamente, objetivándose en el fondo la FAVI (Fig.2), por lo que además se asoció al tratamiento Gentamicina vía parenteral. Se continuó curando con Aquacel $\mathrm{Ag}^{\circledR}$, cada dos días; en la tercera cura, al retirar el apósito, la herida presentaba mucho peor aspecto (Fig.3), desprendía mal olor, por lo que se volvió a tomar muestra para cultivo del exudado. Se limpió y lavó con suero salino 0,9\% y comenzamos a curar con pomada desbridante con enzimas proteolíticos, principalmente colagenasa (Iruxol ${ }^{\circledR}$ Mono).

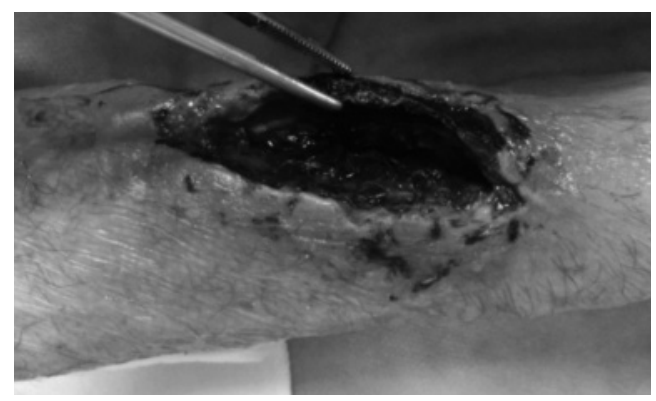

Fig. 2. Herida de grandes dimensiones. FAVI en fondo de herida.

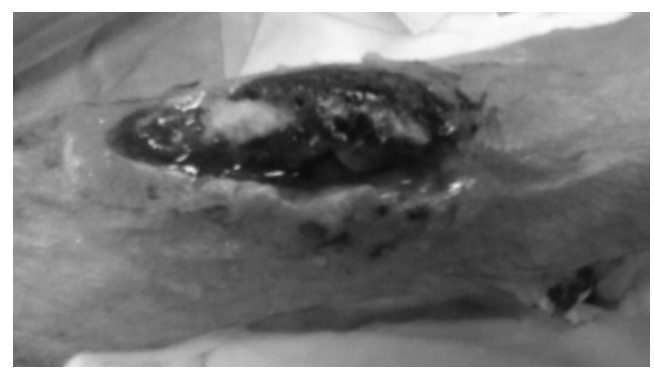

Fig. 3. Herida infectada por Fusobacterium varium.

Apareció en el exudado un germen Gram negativo, Fusobacterium varium, sensible a Metronidazol, por lo que se suspendió la antibioterapia intravenosa y se administró este fármaco en dosis de $500 \mathrm{mg} / 6 \mathrm{~h}$, por vía oral.

Varios días después observamos que comienza a aparecer en el lecho de la herida tejido de granulación (Fig.4), por lo que pasamos a curarlo con apósitos de alginato cálcico con plata (Algisite $\mathrm{Ag}^{\circledR}$ ). La herida va mejorando visiblemente a medida que fuimos realizando las curas. Cuando estaba prácticamente epitelizada comenzamos a curarla con apósitos de espuma de poliuretano absorbente con Tecnología Safetac. (Mepilex ${ }^{\circledR}$ ) (Fig.5). 


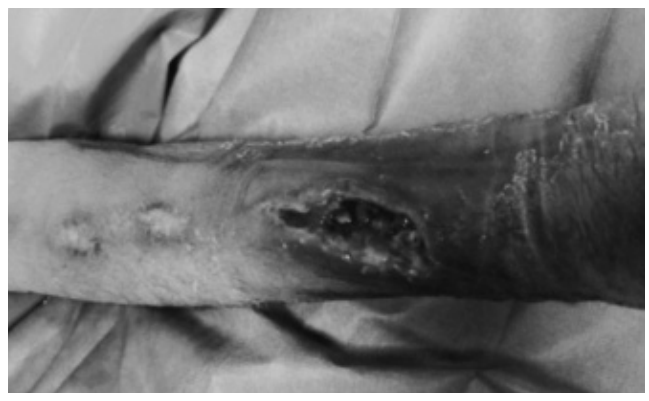

Fig. 4. Herida con tejido de granulación.

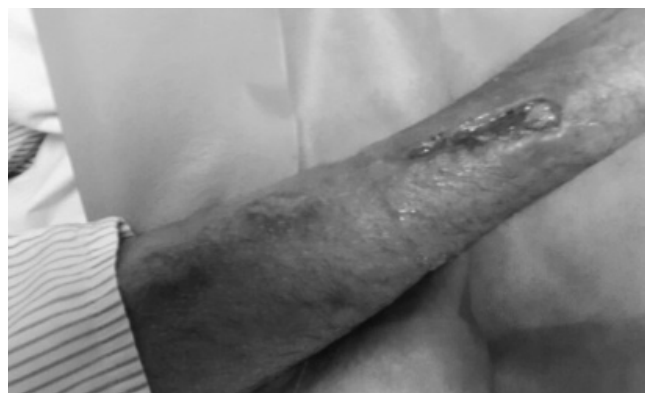

Fig. 5. Herida con evolución favorable.

Días más tarde al realizar la cura observamos emergiendo de la herida un hilo de sutura (Fig.6), que al intentar retirarlo notamos que tiraba de "algo interno" por lo que volvemos a enviarle a cirugía vascular, dado el proceso que había pasado. Allí lo retiraron.

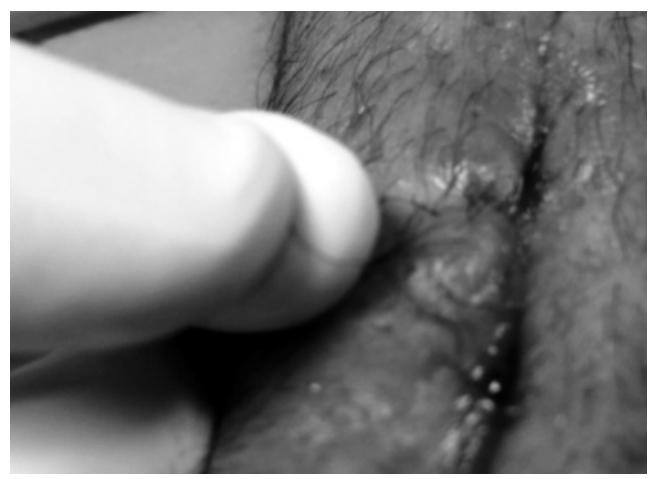

Fig. 6. Sutura emergiendo por la herida.

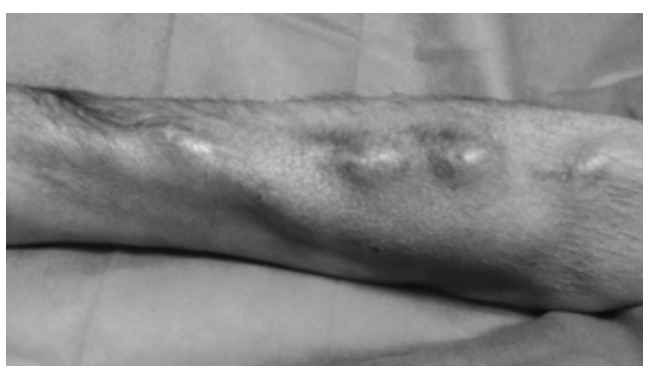

Fig. 7. Herida completamente curada.
Por nuestra parte seguimos curando con los apósitos de Mepilex ${ }^{\circledR}$ hasta que cerró completamente (Fig.7).

Decidimos dejar pasar unos días antes de comenzar a utilizar la FAVI, que presentaba buen thrill, como acceso vascular, dado que el CVCT permitía buenos flujos y unas HD de calidad. Éstas se realizaron inicialmente a través de ambos accesos vasculares para pasar en dos o tres sesiones a utilizar dicha FAVI como único acceso vascular, obteniendo buenos flujos que fuimos aumentando gradualmente: $320-350-400$ $\mathrm{ml} / \mathrm{min}$.

\section{Aspectos éticos}

Los autores declaran que el paciente manifestó su conformidad con la presentación del estudio, así como que no existe conflicto de intereses.

\section{Resultados}

Gracias a la vigilancia periódica del AV por enfermería se pusieron en marcha, precozmente, las estrategias necesarias para resolver las dos primeras complicaciones. No se pudo evitar la infección, pero sí lograr, tras las curas aplicadas, eliminar la solución de continuidad consiguiendo una cicatrización correcta de tejidos adyacentes y piel. Una vez que la herida ha estado cerrada hemos vuelto a realizar HD a través de la FAVI obteniendo flujos que nos han permitido unas HD adecuadas.

\section{Discusión}

Los accesos vasculares requieren para su realización, mantenimiento y supervivencia de la implicación de un equipo multidisciplinar. El poder disponer de un acceso vascular adecuado va a condicionar la calidad de la HD $y$, por ende, la calidad de vida del paciente. Conscientes de este hecho, desde la Sociedad Española de Nefrología (SEN) se impulsó la creación de las "Guías de acceso vascular para HD" implicando en ellas a miembros de otras sociedades profesionales tales como la de Radiología Vascular, de Enfermedades Infecciosas y Microbiología, de Angiología y Cirugía Vascular y la Sociedad Española de Enfermería Nefrológica.

Dentro de este grupo, la enfermería nefrológica tiene un papel primordial pues es quien inspecciona, maneja y cuida, en cada sesión, el AV que porta el paciente, siguiendo los protocolos de monitorización y vigilancia 
del mismo que deben existir en todas las unidades de HD. De esta manera, en caso de complicaciones, se puede poner en marcha, rápidamente, la estrategia necesaria para solucionarlas, pues algunas requieren una intervención inmediata (no más de 48 horas), como ocurre en el caso de trombosis ${ }^{1}$. Además hemos de tener en cuenta también lo que algunos autores denominan "factor centro", es decir, no todos los hospitales ni centros de diálisis disponen de un equipo de cirugía vascular, como ocurre en nuestro caso, de ahí que sea tan importante detectar precozmente las complicaciones, así como tener bien definido el proceso de derivación a otros miembros del equipo multidisciplinar.

Respecto a la infección de la herida, al paciente se le fue administrando el tratamiento antibiótico pautado por el nefrólogo responsable, según resultados microbiológicos. Por parte de enfermería, fuimos realizando las curas con antisépticos como la povidona yodada (Betadine $\left.{ }^{\circledast}\right)^{4}$, apósitos como Aquace ${ }^{\circledR} \mathrm{Ag}^{5}$ primero, tal como vimos en algún estudio ${ }^{6}$, pomadas desbridantes como Iruxol ${ }^{\circledR}$ Mono $^{7}$ después, para volver a aplicar nuevos apósitos como el Algisite ${ }^{\circledR} \mathrm{Ag}^{8}$ y Mepilex ${ }^{\circledR 9}$. Realizamos las curas en función de la evolución de la herida con los productos que, junto con el nefrólogo y el cirujano vascular, creímos más convenientes y de los que podíamos disponer en nuestro centro de trabajo. Gracias a tan estrecha colaboración, pudimos llevar a buen fin el rescate de este AV.

\section{Conclusiones}

La detección precoz de las complicaciones del AV por enfermería, permitió una rápida intervención del nefrólogo y del cirujano vascular, que unidas a la prestación de unos cuidados de enfermería adecuados (seguimiento y curas durante la infección) nos han permitido el rescate de un acceso vascular que, a priori, parecía bastante difícil.

Una vez más, se demuestra que es necesaria e imprescindible la colaboración de todos los miembros del equipo multidisciplinar para la realización, seguimiento y mantenimiento de los accesos vasculares para HD.

\section{Agradecimientos}

A todo el personal de enfermería que participó en las curas.

A la Dra. Belén Gómez Giralda, Facultativo Especialista de Área de Nefrología del Hospital Universitario Río Hortega de Valladolid.
Al Dr. Álvaro Revilla Calavia, Facultativo Especialista de Área de Cirugía Vascular del Hospital Clínico Universitario de Valladolid.

Al paciente y esposa por su colaboración en la obtención de las fotografías.

Recibido: 18 octubre 2015

Revisado: 2 noviembre 2015

Modificado: 6 noviembre 2015

Aceptado: 6 noviembre 2015

\section{Bibliografía}

1. Rodríguez JA, González E, Gutiérrez JM, et al. Guías de acceso vascular en hemodiálisis (Guías S.E.N.). Nefrología 2005; 25 (Supl 1):3-97.

2. National Kidney Foundation. KDOQI Clinical Practice Guidelines and Clinical Practice Recommendations for 2006 Updates: Hemodialysis Adequacy, Peritoneal Dialysis Adequacy and Vascular Access. Am J Kidney Dis 2006 (Suppl 1); 48: S1-S322.

3. Solozábal CA. Utilización de catéteres y tipos. En: González MT, Martínez R. Manual de accesos vasculares para hemodiálisis. Valencia: Marge; 2010; p. 75-87.

4. Ficha técnica de Betadine ${ }^{\circledR}$, disponible en: www.infomecum.es

5. Ficha técnica de Aquacel $\mathrm{Ag}^{\circledR}$, disponible en: www.infomecum.es

6. Rodríguez C. Canals M.E. De Juan J. Tratamiento de una herida quirúrgica próxima a una fístula arterio-venosa con Aquacel® Ag. Rev Soc Esp Enferm Nefrol 2008; 11 (3): 242/245.

7. Ficha técnica de Iruxol ${ }^{\circledR}$ Mono, disponible en: www.infomecum.es

8. Ficha técnica de Algisite $\mathrm{Ag}^{\circledR}$, disponible en: www.infomecum.es

9. Ficha técnica de Mepilex ${ }^{\circledR}$, disponible en: www.infomecum.es 